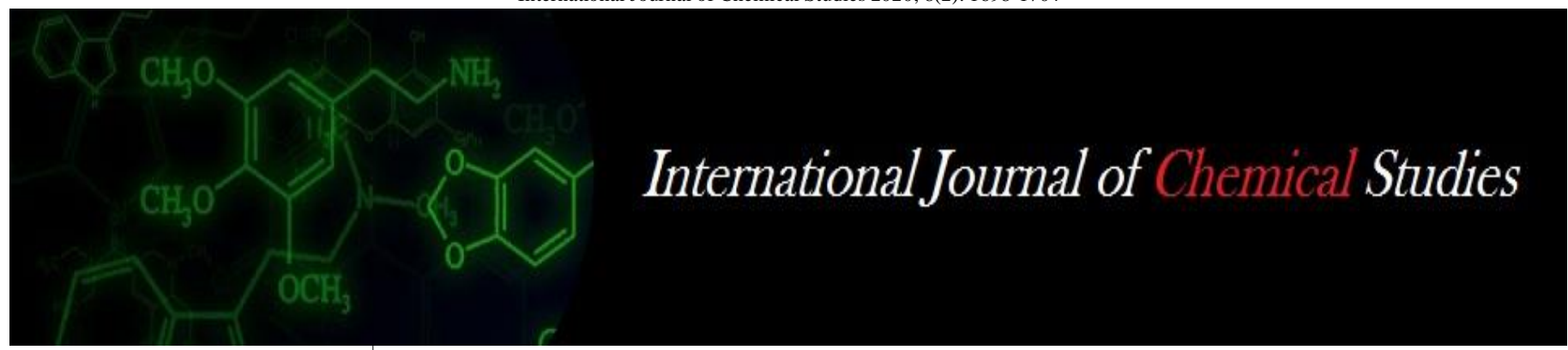

P-ISSN: 2349-8528

E-ISSN: 2321-4902

www.chemijournal.com

IJCS 2020; 8(2): 1698-1704

(C) 2020 IJCS

Received: 24-01-2020

Accepted: 26-02-2020

Khonde SS

M.Sc. Agri. (Plant Physiology),

PGI, Dr. Punjabrao Deshmukh

Krishi Vidyapeeth, Akola,

Maharashtra, India

Phad DS

Assistant vegetable breeder

chilli and vegetable research unit

Dr. Punjabrao Deshmukh Krishi

Vidyapeeth, Akola,

Maharashtra, India

Bharad SG

Head, Wheat research unit Dr.

Panjabrao Deshmukh Krishi

Vidyapeeth, Akola,

Maharashtra, India

Kayande NV

Assistant professor,

Department of Agril. botany

PGI, Dr. Punjabrao Deshmukh

Krishi Vidyapeeth, Akola,

Maharashtra, India

Corresponding Author:

Khonde SS

M.Sc. Agri. (Plant Physiology),

PGI, Dr. Punjabrao Deshmukh

Krishi Vidyapeeth, Akola,

Maharashtra, India

\section{Physiological evaluation of wheat genotypes under normal irrigated and non-irrigated conditions}

\author{
Khonde SS, Phad DS, Bharad SG and Kayande NV
}

DOI: https://doi.org/10.22271/chemi.2020.v8.i2z.9007

\begin{abstract}
The present inves Stigation entitled as "Physiological evaluation of wheat genotype under normal irrigated and non-irrigated conditions" was carried out in rabi season of 2017-18 at Wheat Research Unit, Dr. Panjabrao Deshmukh Krishi Vidyapeeth, Akola (M.S.). It is carried out under two sowing environment i.e. normal irrigated and non-irrigated condition and 25 wheat genotypes viz., HD 3237, DBW 166, JWS 810, RIL-S1-126, DBW 110, DBW 93, WH 1235, MP 3288, GW 477, NIAW 3170, K 1317, DBW 136, HI 1620, M 516, DBW 252, NI 5439, RIL-S1-38, MACS 6696, RW 5, NIAW 3212, BRW 3806, C 306, MACS 6695, HI 1628, MP 133 were tested. The experiment was laid out in Simple lattice design with two replications.

Different yield and physiological traits viz. germination, days to heading, days to anthesis, days to maturity, plant height, number of productive tillers at maturity, number of grains per spike, grain weight/ spike, 1000 grain weight, biological yield, grain yield, harvest index, chlorophyll content index (at 15 and 21 days after anthesis), canopy temperature (at 15 and 21 days after anthesis) may be used in research programme for development of moisture tolerant genotypes for changing climatic scenario.

The growth, yield attributes and physiological attributes showed significant increase when wheat crop was sown under normal irrigated condition. Grain yield obtained was significantly higher in sowing under irrigated condition. Wheat crop sown at irrigated condition showed lowest canopy temperature (at 15 and 21 days after anthesis) as compare to sown under non-irrigated condition. Sowing of wheat crop under irrigated condition was found to be most economical.

Out of 25 genotypes tested under experiment, genotype GW 477 was superior over other in respect of number of productive tillers, number of grains per spike, grain weight/ spike, grain yield, harvest index as compare to other genotypes under normal irrigated condition. In non-irrigated condition, MACS 6696 superior in respect of grain yield, harvest index and chlorophyll content index at 21 days after anthesis
\end{abstract}

Keywords: Physiological evaluation, normal irrigated and non-irrigated conditions

\section{Introduction}

Wheat is grown in all the states in India except Southern and North Eastern states. Uttar Pradesh, Haryana, Punjab, Rajasthan are the major wheat producing states and accounts for almost $80 \%$ of total production in India. Only $13 \%$ area is rainfed, Major rainfed wheat area found in Madhya Pradesh, Gujarat, Maharashtra, West Bengal and Karnataka. Central and Peninsular Zone accounts total $1 / 3^{\text {rd }}$ of wheat area in India. On all India basis, only $1 / 3$ irrigated wheat receives desired irrigations and remaining is limited irrigation only. Breeding programmes are generally aimed for rainfed and irrigated environments and there is need to develop varieties which are responsive to limited irrigation conditions. Thus, to increase the productivity of this region different physiological technique need to be adopted for improving water use efficiency and breeding wheat genotypes tolerant to water stress.

\section{Material and methods}

The present investigation entitled that, "Physiological evaluation of wheat genotypes under normal irrigated and non-irrigated condition" laid out in simple lattice design with full irrigated and non-irrigated environments as a 5 block and 25 genotypes. 
Treatment details.

\begin{tabular}{|l|c|c|c|c|c|}
\hline \multicolumn{7}{|c|}{ Replication 1 } \\
\hline Block 1 & HD 3237 & DBW 166 & JWS 810 & RIL-S1-126 & DBW 110 \\
\hline Block 2 & DBW 93 & WH 1235 & MP 3288 & GW 477 & NIAW 3170 \\
\hline Block 3 & K 1317 & DBW 136 & HI 1620 & M 516 & DBW 252 \\
\hline Block 4 & NI 5439 & RIL-S1-38 & MACS 6696 & RW 5 & NIAW 3212 \\
\hline Block 5 & BRW 3806 & C 306 & MACS 6695 & HI 1628 & MP 1331 \\
\hline \multicolumn{7}{|c|}{ Replication 2 } & DBW 110 & NI 5439 \\
\hline Block 1 & M 516 & MP 3288 & C 306 & RIL-S1-38 & HI 1620 \\
\hline Block 2 & MP 1331 & RIL-S1-126 & DBW 93 & GW 477 & DBW 166 \\
\hline Block 3 & MACS 6696 & BRW 3806 & DBW 252 & MACS 6695 & NIAW 3170 \\
\hline Block 4 & HD 3237 & DBW 136 & RW 5 & HI 1628 & MP 1331 \\
\hline Block 5 & BRW 3806 & C 306 & MACS 6695 &
\end{tabular}

\section{Results and discussion}

The results obtained are discussed to have a clear understanding about the relationship of different factors viz. irrigated and non-irrigated condition on morphological and physiological characters of wheat genotypes.

\section{Yield parameters}

1.1 Germination $(\%)$ after two weeks of sowing.
Numerically out of 25 genotypes only one genotype K 1317 $(98.61 \%)$ recorded statistical significance over other genotypes and remaining all genotype at par with general mean and with each other however under non-irrigated condition all genotypes at par with general mean and with each other for germination. Highest germination (\%) was recorded in genotype NIAW $3170(95.03 \%)$ under nonirrigated condition.

Table 1: Mean performance of different genotypes under normal irrigated and non-irrigated condition for germination (\%) and days to heading $(75 \%)$.

\begin{tabular}{|c|c|c|c|c|}
\hline \multirow{2}{*}{ Treatment } & \multicolumn{2}{|c|}{ Germination \% } & \multicolumn{2}{|c|}{ Days to heading $(75 \%)$} \\
\hline & Normal irrigated & Non-irrigated & Normal irrigated & Non-irrigated \\
\hline BRW 3806 & 93.32 & 92.51 & 67.58 & 69.53 \\
\hline DBW 110 & 93.37 & 85.08 & 68.01 & 67.39 \\
\hline DBW 136 & 88.98 & 90.01 & 68.82 & $60.89 *$ \\
\hline DBW 166 & 90.94 & 84.98 & 69.01 & $63.93 *$ \\
\hline DBW 252 & 92.91 & 82.61 & 68.32 & 66.35 \\
\hline GW 477 & 89.8 & 87.56 & 64.99 & 64.74 \\
\hline HD 3237 & 90.07 & 87.6 & 66.32 & 64.08 \\
\hline HI 1620 & 91.14 & 84.48 & 64.23 & 64.64 \\
\hline HI 1628 & 88.59 & 87.52 & 66.32 & 65.95 \\
\hline JWS 810 & 95.61 & 91.44 & 62.42 & 64.17 \\
\hline M 516 & 88.37 & 85.15 & 67.88 & 65.46 \\
\hline MACS 6695 & 92.86 & 87.35 & 66.68 & $62.62 *$ \\
\hline MACS 6696 & 96.03 & 82.34 & $59.61 *$ & 67.45 \\
\hline MP 1331 & 93.13 & 84.98 & 65.26 & $61.84 *$ \\
\hline NIAW 3170 & 94.34 & 95.03 & 67.43 & $62.14 *$ \\
\hline NIAW 3212 & 89.8 & 84.97 & 67.69 & 64.66 \\
\hline RIL-S1-126 & 90.34 & 90.11 & 65.07 & 68.5 \\
\hline RIL-S1-38 & 88.37 & 87.37 & 67.19 & 68.2 \\
\hline RW 5 & 86.76 & 82.51 & $60.25 *$ & $60.27 *$ \\
\hline WH 1235 & 90.4 & 89.93 & 64.93 & 64.67 \\
\hline DBW 93(C) & 97.53 & 87.56 & 69.25 & 70.83 \\
\hline MP 3288(C) & 96.57 & 89.90 & 68.35 & 64.42 \\
\hline K 1317(C) & 98.61 & 80.14 & 69.63 & 67.54 \\
\hline NI 5439(C) & 96.99 & 92.50 & 68.51 & 64.85 \\
\hline C 306(C) & 95.69 & 93.38 & 68.76 & 67.87 \\
\hline GM & 92.42 & 87.48 & 66.50 & 65.32 \\
\hline $\mathrm{SE}(\mathrm{m}) \pm$ & 1.96 & 3.85 & 1.88 & 2.44 \\
\hline CD at $5 \%$ & 5.49 & 10.79 & 5.27 & 6.85 \\
\hline "F" test & SIG & SIG & SIG & SIG \\
\hline
\end{tabular}

*- Level of significance at $5 \%$

\subsection{Days to heading (75\%).}

Data indicated that some cultivars headed earlier than others which were in conformity with the findings of Rafiullah et al. (2007). Among the genotypes significant variations was observed under irrigated condition in days to $75 \%$ heading. Significantly minimum days recorded for $75 \%$ heading was observed in two genotypes over check viz. MACS 6696 (59.61) and RW 5 (60.25) However, under non-irrigated condition six genotypes shows statistical significance over check for days to early heading. Significantly minimum days were recorded for $75 \%$ heading was observed in viz. RW 5
(60.27), DBW 136 (60.89) and NIAW 3170 (62.14) respectively.

1.3 Days to maturity $(75 \%)$.

Under normal irrigated condition, five genotypes recorded significantly minimum days to maturity over check. Early days to $75 \%$ maturity were recorded by genotypes MACS 6696 (109.02), RW 5 (110.82) and MACS 6695 (112.38). However under non-irrigated condition, four genotypes recorded statistical significance over check C 306 for days to 75\% maturity. Genotype DBW 110 recorded lowest days to 
$75 \%$ maturity followed by genotypes NIAW 3170 (110.59) and HD 3237 (110.91) under non-irrigated condition. High moisture stress induced by rainfed sowing caused reduction by 1 day for days to maturity as compared to irrigated condition. Similar results were reported by Mekkei et al. (2014) ${ }^{[7]}$, Ngwako and Mashiqa (2013) ${ }^{[9]}$.

4.1.4 Number of productive tillers per meter row length.
Data presented in Table 4.2 indicated that, significant differences in respect to no. of effective tillers per meter at maturity. High moisture stress induced by sowing under nonirrigated condition (rainfed) condition (39.90) significantly decreased number of effective tillers per meter at maturity as compared to sown under irrigated

Table 2: Mean performance of different genotypes under normal irrigated and non-irrigated condition for days to maturity (75\%) and productive tillers/ meter row.

\begin{tabular}{|c|c|c|c|c|}
\hline \multirow[b]{2}{*}{ Treatment } & \multicolumn{2}{|c|}{ Days to maturity (75\%) } & \multicolumn{2}{|c|}{ Productive tillers/ meter row } \\
\hline & Normal irrigated & Non-irrigated & Normal irrigated & Non-irrigated \\
\hline BRW 3806 & 114.97 & 118.91 & 63.41 & 38.43 \\
\hline DBW 110 & 118.42 & 116.76 & 62.45 & 39.15 \\
\hline DBW 136 & 117.56 & $110.5^{*}$ & 61.74 & 36.67 \\
\hline DBW 166 & 119.89 & 113.86 & 61.85 & 38.83 \\
\hline DBW 252 & 118.59 & 116.4 & 61.84 & 37.50 \\
\hline GW 477 & 114.53 & 113.9 & 73.84* & 38.3 \\
\hline HD 3237 & 117.03 & $110.91 *$ & 63.54 & 39.33 \\
\hline HI 1620 & $112.61 *$ & 114.24 & 62.69 & 37.98 \\
\hline HI 1628 & 117.18 & 115.56 & 61.15 & 38.96 \\
\hline JWS 810 & 118.94 & 114.1 & $72.30 *$ & 40.13 \\
\hline M 516 & 118.91 & 115.7 & 60.68 & 40.70 \\
\hline MACS 6695 & $112.38^{*}$ & $112.1^{*}$ & 62.16 & 36.24 \\
\hline MACS 6696 & $109.02 *$ & 116.94 & 65.57 & 37.44 \\
\hline MP 1331 & 116.36 & 117.26 & 62.81 & 41.26 \\
\hline NIAW 3170 & 117.71 & $110.59 *$ & $73.00 *$ & 41.61 \\
\hline NIAW 3212 & 118 & 114.09 & 62.72 & 39.96 \\
\hline RIL-S1-126 & 115.74 & 117.06 & 63.28 & 39.35 \\
\hline RIL-S1-38 & 117.97 & 118.2 & 61.62 & 44.13 \\
\hline RW 5 & $110.82 *$ & 114.4 & 61.55 & 36.65 \\
\hline WH 1235 & $113.18^{*}$ & 113.2 & 62.90 & 42.28 \\
\hline DBW 93(C) & 119.32 & 119.74 & 75.60 & 39.28 \\
\hline MP 3288(C) & 116.73 & 113.44 & 76.85 & 43.59 \\
\hline K 1317(C) & 119.2 & 116.55 & 74.93 & 38.67 \\
\hline NI 5439(C) & 115.11 & 115.24 & 68.81 & 43.63 \\
\hline C 306(C) & 119.83 & 119.86 & 74.22 & 47.43 \\
\hline GM & 116.4 & 115.18 & 66.06 & 39.90 \\
\hline $\mathrm{SE}(\mathrm{m}) \pm$ & 2.28 & 2.41 & 0.91 & 2.41 \\
\hline $\mathrm{CD}$ at $5 \%$ & 6.41 & 6.76 & 2.56 & 6.75 \\
\hline "F" test & SIG & SIG & SIG & SIG \\
\hline
\end{tabular}

*- Level of significance at $5 \%$

condition (66.06) in wheat crop. Similar results were reported by Mekkei et al. (2014) ${ }^{[7]}$.

\subsection{Plant height (cm).}

Data on plant height $(\mathrm{cm})$ was recorded at harvest presented in table 4.3. From data it was observed that, plant height progressively increase in different genotypes. It indicated that there was significant difference in plant height.

Data pertaining to plant height at harvest revealed that, among the 25 wheat genotypes, eight genotypes shows statistical significance over check. DBW 166 recorded significantly lowest plant height $(80.96 \mathrm{~cm})$ at harvest and followed by DBW $136(80.99 \mathrm{~cm})$ and MP 1331 $(80.99 \mathrm{~cm})$. Under non-irrigated condition, 17 genotypes recorded significantly lowest plant height at harvest. Genotype GW 477 showed significantly lowest plant height $(43.12 \mathrm{~cm})$ at harvest followed by DBW $252(45.59 \mathrm{~cm})$ and MACS 6696 $(46.5 \mathrm{~cm})$. Genotype C 306 recorded highest plant height under both irrigated and non-irrigated condition.

\subsection{0 grain weight $(\mathrm{g})$.}

Table 3: Mean performance of different genotypes under normal irrigated and non-irrigated condition for plant height $(\mathrm{cm})$ and 1000 grain weight.

\begin{tabular}{|c|c|c|c|c|}
\hline \multirow{2}{*}{ Treatment } & \multicolumn{2}{|c|}{ Plant height (cm) } & \multicolumn{2}{c|}{ 1000 grain weight } \\
\cline { 2 - 5 } & Normal irrigated & Non-irrigated & Normal irrigated & Non-irrigated \\
\hline BRW 3806 & 86.00 & $58.46^{*}$ & $52.59^{*}$ & $41.29^{*}$ \\
\hline DBW 110 & 83.97 & 66.58 & 47.13 & 30.79 \\
\hline DBW 136 & $80.99^{*}$ & $59.74^{*}$ & 45.77 & 33.03 \\
\hline DBW 166 & $80.96^{*}$ & $52.24^{*}$ & 49.1 & 23.04 \\
\hline DBW 252 & 93.00 & $45.59^{*}$ & 43.9 & 29.62 \\
\hline GW 477 & 86.00 & $43.21^{*}$ & $50.08^{*}$ & $38.44^{*}$ \\
\hline
\end{tabular}




\begin{tabular}{|c|c|c|c|c|}
\hline HD 3237 & 84.97 & $56.85 *$ & 47.33 & 27.28 \\
\hline HI 1620 & $81.05 *$ & $55.93 *$ & $54.19 *$ & $41.80 *$ \\
\hline HI 1628 & 88.01 & 64.24 & 46.46 & 34 \\
\hline JWS 810 & 86.52 & $56.93 *$ & 46.65 & 25.8 \\
\hline M 516 & $83.01 *$ & $59.64 *$ & 41.91 & 32.67 \\
\hline MACS 6695 & $83.55 *$ & $56.54 *$ & $52.53 *$ & 29.81 \\
\hline MACS 6696 & 84.54 & $46.5^{*}$ & 46.46 & 31.5 \\
\hline MP 1331 & $80.99 *$ & $59.7 *$ & 48.86 & 37.98 \\
\hline NIAW 3170 & 87.99 & 63.67 & 39.58 & $40.00 *$ \\
\hline NIAW 3212 & $81.99 *$ & $55.66 *$ & $51.22 *$ & 33.23 \\
\hline RIL-S1-126 & 89.48 & $52.13 *$ & 47.36 & 25.24 \\
\hline RIL-S1-38 & $81.98 *$ & $59.81 *$ & $52.89 *$ & 31.62 \\
\hline RW 5 & 83 & $54.2 *$ & 49.67 & 35.09 \\
\hline WH 1235 & 82.48 & $58.32 *$ & 47.88 & 29.73 \\
\hline DBW 93(C) & 80.99 & 43.93 & 52.60 & 34.27 \\
\hline K 1317(C) & 90.01 & 59.35 & 53.97 & 41.26 \\
\hline NI 5439(C) & 86.99 & 70.42 & 52.57 & 31.13 \\
\hline C 306(C) & 111.98 & 81.35 & 54.17 & 40.28 \\
\hline GM & 85.88 & 57.54 & 48.88 & 33.51 \\
\hline $\mathrm{SE}(\mathrm{m}) \pm$ & 2.26 & 7.23 & 0.91 & 2.51 \\
\hline $\mathrm{CD}$ at $5 \%$ & 6.35 & 20.29 & 2.56 & 7.03 \\
\hline "F" test & SIG & SIG & SIG & SIG \\
\hline
\end{tabular}

*- Level of significance at $5 \%$

Under non-irrigated condition, four genotypes shows statistical significance over check. Genotype HI 1620 (41.80 g) recorded highest 1000 grain weight and followed BRW 3806 (41.30 g), NIAW 3170 (40 g) and GW 477 (38.44 g). Lowest 1000 grain weight was recorded in DBW 166 (23.04 g).

\subsection{Number of grains spike ${ }^{-1}$.}

Data pertaining to number of grains spike ${ }^{-1}$ were presented in table 4.4 indicated that, number of grains spike ${ }^{-1}$ were reduced under non-irrigated condition (rainfed) condition (27.32) as compared to normal irrigated condition (43.98).It might be due to favourable moisture regime helped in proper growth and development of crop that reflected in higher number of grains per spike. Similar results were reported by Khan et al. (2007), Akbari et al. (2011) ${ }^{[1]}$.

Table 4: Mean performance of different genotypes under normal irrigated and non-irrigated condition for Number of grains/ spike and grain weight/ spike

\begin{tabular}{|c|c|c|c|c|}
\hline \multirow{2}{*}{ Treatment } & \multicolumn{2}{|c|}{ No. of grains/ spike } & \multicolumn{2}{|c|}{ Grain weight/spike } \\
\hline & Normal irrigated & Non-irrigated & Normal irrigated & Non-irrigated \\
\hline BRW 3806 & 43.67 & $33.50 *$ & 3.55 & 1.45 \\
\hline DBW 110 & 43.41 & 26.39 & 3.28 & 1.65 \\
\hline DBW 136 & $53.44 *$ & 25.69 & 3.09 & 1.80 \\
\hline DBW 166 & $46.08 *$ & 29.99 & 3.15 & 0.70 \\
\hline DBW 252 & $55.27 *$ & 28.1 & 2.91 & 1.50 \\
\hline GW 477 & $54.71 *$ & 24.47 & $3.86^{*}$ & 1.35 \\
\hline HD 3237 & $50.85 *$ & 23.42 & 3.15 & 0.95 \\
\hline HI 1620 & $46.59 *$ & 30.18 & $3.87 *$ & 1.10 \\
\hline HI 1628 & 39.44 & 28.47 & 3.38 & 1.15 \\
\hline JWS 810 & 39.23 & 24.47 & 2.85 & 0.70 \\
\hline M 516 & 42.48 & 26.6 & 2.70 & 1.80 \\
\hline MACS 6695 & 41.06 & 25.55 & 3.54 & 1.55 \\
\hline MACS 6696 & 41.27 & 28.95 & 3.02 & 1.65 \\
\hline MP 1331 & 35.23 & 28.47 & 2.90 & 1.15 \\
\hline NIAW 3170 & 44 & 26.47 & 3.58 & 0.50 \\
\hline NIAW 3212 & $50.94 *$ & 26.86 & 3.44 & 1.70 \\
\hline RIL-S1-126 & 42.12 & 23.89 & 3.25 & 1.05 \\
\hline RIL-S1-38 & 45.12 & 25.46 & 2.72 & 1.40 \\
\hline RW 5 & 43.15 & 28.86 & 3.52 & $2.00 *$ \\
\hline WH 1235 & $48.67 *$ & 27.57 & 3.28 & 1.35 \\
\hline DBW 93(C) & 35.44 & 27.50 & 3.68 & 1.50 \\
\hline MP 3288(C) & 35.33 & 26.55 & 3.23 & 1.35 \\
\hline K 1317(C) & 35.21 & 30.12 & 4.11 & 1.80 \\
\hline NI 5439(C) & 36.88 & 27.89 & 3.59 & 1.80 \\
\hline C 306(C) & 49.91 & 27.57 & 4.14 & 1.40 \\
\hline GM & 43.98 & 27.32 & 3.35 & 1.37 \\
\hline $\mathrm{SE}(\mathrm{m}) \pm$ & 1.40 & 1.56 & 0.20 & 0.21 \\
\hline $\mathrm{CD}$ at $5 \%$ & 3.92 & 4.38 & 0.57 & 0.59 \\
\hline "F" test & SIG & SIG & SIG & SIG \\
\hline
\end{tabular}

*- Level of significance at $5 \%$

1.8 Grain weight/ spike (g). 
The data presented in table 4.4 revealed that, Grain weight/ spike (g) was found maximum under normal irrigated condition (4.14 g) in relation to non-irrigated (rainfed) condition $(2.00 \mathrm{~g})$ in wheat. The reduction in Grain weight/ spike was caused due to high moisture stress induced by nonirrigated (rainfed) condition. Similar results were reported by Hasan et al. (2010).

\subsection{Grain yield ( $\mathrm{Kg} / \mathrm{ha})$.}

Under normal irrigated condition, only one genotype GW 477 $(3640.6 \mathrm{Kg} / \mathrm{ha})$ showed statistical significance for grain yield $\left(\mathrm{Kg}\right.$ plot $\left.^{-1}\right)$ over check. In case of non-irrigated condition,
Genotype MACS $6696(2130.91 \mathrm{Kg} / \mathrm{ha})$ recorded the significantly highest grain yield. Genotypic differences in grain yield might be due to genetic potential of different varieties to express in terms yield attributing traits in differential sowing environment conditions.

\subsection{Biological yield $\left(\mathrm{Kg} \mathrm{plot}^{-1}\right)$.}

The data pertaining to biological yield $\left(\mathrm{Kg}_{\mathrm{glot}}{ }^{-1}\right)$ presented in table 4.5. However, numerically maximum biological yield was recorded in normal irrigated condition $\left(0.64 \mathrm{Kg}_{\text {plot }}{ }^{-1}\right)$ and minimum in non-irrigated condition (rainfed) condition.

Table 5: Mean performance of different genotypes under normal irrigated and non-irrigated condition for grain yield (kg/ha) and biomass (kg/plot).

\begin{tabular}{|c|c|c|c|c|}
\hline \multirow{2}{*}{ Treatment } & \multicolumn{2}{|c|}{ Grain yield (Kg/ha) } & \multicolumn{2}{|c|}{ Biomass (Kg/ plot) } \\
\hline & Normal Irrigated & Non-Irrigated & Normal Irrigated & Non-Irrigated \\
\hline BRW 3806 & 2612.2 & 1385 & 0.62 & 0.30 \\
\hline DBW 110 & 2295.3 & 1227.5 & 0.63 & 0.32 \\
\hline DBW 136 & 2764.2 & 1777.8 & 0.54 & 0.37 \\
\hline DBW 166 & 2663.8 & 1276.8 & 0.62 & 0.38 \\
\hline DBW 252 & 2262.4 & 1417.4 & 0.58 & 0.34 \\
\hline GW 477 & $3640.6 *$ & 1061.8 & 0.67 & 0.24 \\
\hline HD 3237 & 2224.1 & 1172.5 & 0.7 & 0.24 \\
\hline HI 1620 & 2316.3 & 1574.9 & 0.54 & 0.34 \\
\hline HI 1628 & 2975.4 & 1474.9 & 0.72 & 0.33 \\
\hline JWS 810 & 2793.8 & 1251.7 & 0.63 & 0.26 \\
\hline M 516 & 2243.2 & 1318.8 & 0.61 & 0.27 \\
\hline MACS 6695 & 2470.7 & $2130.9 *$ & 0.53 & $0.42 *$ \\
\hline MACS 6696 & 3132.1 & 1347.8 & 0.65 & 0.30 \\
\hline MP 1331 & 2594.5 & 1395.6 & 0.56 & 0.39 \\
\hline NIAW 3170 & 3126.4 & 1293.7 & 0.76 & 0.30 \\
\hline NIAW 3212 & 2144.8 & 1234.8 & 0.52 & 0.29 \\
\hline RIL-S1-126 & 2054.0 & 1617.9 & 0.46 & 0.35 \\
\hline RIL-S1-38 & 2646.6 & 1728.5 & 0.64 & $0.47 *$ \\
\hline RW 5 & 2570.1 & 1491.8 & 0.52 & 0.38 \\
\hline WH 1235 & 2739.3 & 1787.4 & 0.52 & $0.43 *$ \\
\hline DBW 93(C) & 4432.98 & 1638.657 & 0.86 & 0.37 \\
\hline MP 3288(C) & 3047.06 & 1451.215 & 0.79 & 0.33 \\
\hline K 1317(C) & 4013.37 & 1806.771 & 0.83 & 0.31 \\
\hline NI 5439(C) & 3540.27 & 1446.383 & 0.84 & 0.38 \\
\hline C 306(C) & 4096.33 & 1622.7 & 0.79 & 0.41 \\
\hline GM & 2856.00 & 1477.334 & 0.64 & 0.34 \\
\hline $\mathrm{SE}(\mathrm{m}) \pm$ & 293.53 & 154.90 & 0.07 & 0.03 \\
\hline $\mathrm{CD}$ at $5 \%$ & 823.48 & 434.55 & 0.21 & 0.09 \\
\hline "F" test & SIG & SIG & SIG & SIG \\
\hline
\end{tabular}

*- Level of significance at $5 \%$.

$\left(0.34 \mathrm{Kg} \mathrm{plot}^{-1}\right)$ in wheat crop. Similar results were reported by Akbari et al. (2011) ${ }^{[1]}$, Aslam et al. (2014) ${ }^{[3]}$, Mobasser et al. $(2014)^{[8]}$.

\section{Physiological parameters}

2.1 Chlorophyll content index (CHL 1) (At 15 days after anthesis).

Data recorded on chlorophyll content index are presented in Table 4.7. It was evident that CCI was significantly increased under normal irrigated condition (47.56) in comparison to non-irrigated (rainfed) condition (42.16). It might be due to loss of chlorophyll content index under water stress is considered a main cause of inactivation of photosynthesis.
Similar results were reported by Taiz and Zeiger (2006), Tas and Tas (2007) ${ }^{[19]}$.

\subsection{Chlorophyll content index (CHL 2) (21 days after anthesis).}

The data regarding chlorophyll content index are presented in table 4.7 revealed that, the CCI was found increased under normal irrigated condition (39.45) as compared to nonirrigated condition (rainfed) condition (16.01) of wheat crop. Similar results were reported by Saeidi and Abdoli (2015) ${ }^{[12]}$, Sheoran et al. (2015) ${ }^{[15]}$.

Genotype MACS 6695 (22.23) recorded highest value for chlorophyll content index at 21 days after anthesis nonirrigated condition. 
Table 6: Mean performance of different genotypes under normal irrigated and non-irrigated condition for Chlorophyll content index at 15 and 21 days after anthesis.

\begin{tabular}{|c|c|c|c|c|}
\hline \multirow{2}{*}{ Treatment } & \multicolumn{2}{|c|}{ Chlorophyll content index at 15 days after anthesis } & \multicolumn{2}{|c|}{ Chlorophyll content index at 21 days after anthesis } \\
\hline & Normal Irrigated & Non-Irrigated & Normal Irrigated & Non-Irrigated \\
\hline BRW 3806 & 42.26 & 40.55 & 41.03 & 16.18 \\
\hline DBW 110 & 42.9 & 41.41 & 41.7 & 16.8 \\
\hline DBW 136 & 45.33 & 42.56 & 30.24 & 13.23 \\
\hline DBW 166 & 51.42 & $45.45^{*}$ & 32.02 & 14.07 \\
\hline DBW 252 & 46.81 & 39.57 & 43.97 & 13.51 \\
\hline GW 477 & 44.29 & $44.55^{*}$ & 41.62 & 14.45 \\
\hline HD 3237 & 48.51 & 41.86 & 26.79 & 13.83 \\
\hline HI 1620 & 51.39 & 41.62 & $47.96^{*}$ & 18.59 \\
\hline HI 1628 & 48.22 & 37.85 & 44.47 & 14.59 \\
\hline JWS 810 & 39.78 & 42.46 & 31.38 & 15.33 \\
\hline M 516 & 46.32 & 38.87 & 40.36 & 14.45 \\
\hline MACS 6695 & 48.43 & 40.86 & 42.03 & $22.23^{*}$ \\
\hline MACS 6696 & 49.02 & $43.97 *$ & 35.01 & 14.23 \\
\hline MP 1331 & 50.16 & 40.51 & 42.99 & 17.66 \\
\hline NIAW 3170 & 45.53 & 41.6 & 39.33 & 14.72 \\
\hline NIAW 3212 & 45.34 & $45.62 *$ & 41.07 & 14.36 \\
\hline RIL-S1-126 & 51.71 & 42.86 & 30.78 & 18.34 \\
\hline RIL-S1-38 & 47.36 & $47.06^{*}$ & 35.09 & 13.03 \\
\hline RW 5 & 47.75 & $44.52 *$ & 37.01 & 14.19 \\
\hline WH 1235 & 47.7 & 37.59 & 31.6 & 13.99 \\
\hline DBW 93(C) & 49.24 & 36.80 & 44.98 & 14.19 \\
\hline MP 3288(C) & 47.66 & 45.50 & 41.07 & 13.34 \\
\hline K 1317(C) & 49.02 & 44.52 & 47.36 & 18.99 \\
\hline NI 5439(C) & 49.64 & 40.62 & 45.11 & 21.23 \\
\hline C 306(C) & 55.47 & 45.24 & 51.31 & 24.73 \\
\hline GM & 47.65 & 42.16 & 39.45 & 16.01 \\
\hline $\mathrm{SE}(\mathrm{m}) \pm$ & 3.02 & 2.51 & 4.24 & 2.30 \\
\hline $\mathrm{CD}$ at $5 \%$ & 8.48 & 7.04 & 11.89 & 6.44 \\
\hline "F" test & SIG & SIG & SIG & SIG \\
\hline
\end{tabular}

\subsection{Canopy temperature $\left({ }^{0} \mathrm{C}\right)$ (at 15 days after anthesis)}

The data pertaining to canopy temperature $\left({ }^{\circ} \mathrm{C}\right)$ are presented in table 4.8 revealed that, increase in canopy temperature was observed with non-irrigated (rainfed) condition $\left(29.04^{\circ} \mathrm{C}\right)$ relative to normal irrigated condition $\left(27.07^{\circ} \mathrm{C}\right) 15$ days after anthesis. Similar results were reported by Siddique et al. (2000) ${ }^{[17]}$.
2.4 Canopy temperature $\left({ }^{0} \mathrm{C}\right)$ (at 21 days after anthesis) The data on canopy temperature at 21 days after anthesis table 4.8 revealed that, canopy temperature was more under non irrigated (rainfed) condition $\left(35.70^{\circ} \mathrm{C}\right)$ compared to normal irrigated condition $\left(34.58^{\circ} \mathrm{C}\right)$. It might be due to under nonirrigated condition stomata close because of reduced water status, leaf temperature rises above ambient air temperature. Similar results were reported by Gautam et al. $(2016)^{[5]}$.

Table 8: Mean performance of different genotypes under normal irrigated and non-irrigated condition for Canopy temperature at 15 and 21 days after anthesis.

\begin{tabular}{|c|c|c|c|c|}
\hline \multirow{2}{*}{ Treatment } & Canopy temperature at 15 days after anthesis & \multicolumn{2}{c|}{ Canopy temperature at 21 days after anthesis } \\
\cline { 2 - 5 } & Normal Irrigated & Non-Irrigated & Normal Irrigated & Non-Irrigated \\
\hline BRW 3806 & 29.74 & $26.46^{*}$ & 36.42 & $34.10^{*}$ \\
\hline DBW 110 & 24.25 & 29.75 & 34.50 & 36.03 \\
\hline DBW 136 & 28.61 & 28.48 & 36.16 & $34.78^{*}$ \\
\hline DBW 166 & 29.25 & 29.71 & 35.25 & 36.42 \\
\hline DBW 252 & 30.16 & 29.37 & 36.61 & $35.49^{*}$ \\
\hline GW 477 & 27.18 & 27.64 & $32.82^{*}$ & $35.47^{*}$ \\
\hline HD 3237 & 26.96 & $26.63^{*}$ & 34.27 & 38.07 \\
\hline HI 1620 & 27.11 & $26.40^{*}$ & 35.74 & $35.64^{*}$ \\
\hline HI 1628 & 29.6 & $29.62^{*}$ & $32.57^{*}$ & $34.73^{*}$ \\
\hline JWS 810 & 25.3 & 31.53 & 36.34 & $34.73^{*}$ \\
\hline M 516 & 30.83 & 28.66 & 34.03 & 36.16 \\
\hline MACS 6695 & 27.93 & 28.81 & $32.68^{*}$ & 36.36 \\
\hline MACS 6696 & 29.97 & 32.27 & $33.55^{*}$ & $34.07^{*}$ \\
\hline MP 1331 & $14.38^{*}$ & 31.58 & 34.45 & 36.06 \\
\hline NIAW 3170 & 26.81 & 28.3 & $31.70^{*}$ & $35.40^{*}$ \\
\hline NIAW 3212 & 28.27 & 32.94 & 35.12 & $32.82^{*}$ \\
\hline RIL-S1-126 & 26.32 & 32.09 & 35.27 & 36.4 \\
\hline RIL-S1-38 & 26.77 & $26.25^{*}$ & 35.37 & $35.06^{*}$ \\
\hline RW 5 & 27.59 & 30.83 & 36.94 & $35.44^{*}$ \\
\hline
\end{tabular}




\begin{tabular}{|c|c|c|c|c|}
\hline WH 1235 & 27.26 & 28.26 & 34.25 & 33.52 \\
\hline DBW 93(C) & 26.68 & 28.23 & 37.33 & 37.34 \\
\hline MP 3288(C) & 28.71 & 27.43 & 33.63 & 35.84 \\
\hline K 1317(C) & 26.92 & 29.45 & 34.84 & 35.20 \\
\hline NI 5439(C) & 24.14 & 28.47 & 31.05 & 37.21 \\
\hline C 306(C) & 26.13 & 26.79 & 34.58 & 35.30 \\
\hline GM & 27.07 & 29.04 & 0.46 & 35.70 \\
\hline SE $(m) \pm$ & 2.72 & 0.93 & SIG & 0.47 \\
\hline CD at 5\% & 7.63 & 2.62 & SIG & SIG \\
\hline "F" test & SIG & & & \\
\hline
\end{tabular}

*- Level of significance at 5\%.

\section{Conclusion}

1. Sowing of wheat crop under normal irrigated condition significantly improved all the morpho-physiological characters viz. no. of productive tillers per meter at maturity, no. of grains spike ${ }^{-1}$, grain weight/ spike, 1000 grain weight, biological yield, grain yield, harvest index, chlorophyll content index (at 15 and 21 days after anthesis), canopy temperature (at 15 and 21 days after anthesis) as compare to sowing of wheat under nonirrigated (rainfed) condition resulted in significantly increase in grain yield of wheat.

2. Among 25 different genotypes, GW 477 recorded significantly higher yield and physiological parameters as compared to any of the genotypes under normal irrigated and under non-irrigated condition, genotype MACS 6696 recorded highest grain yield and chlorophyll content index.

\section{References}

1. Akbari M, Galavi HM, Fanaei HR, Koohkan SHA, Poodineh O. Effect of deficit irrigation on grain yield and some morphological traits of wheat cultivars in drought prone conditions Int. J. Agri. Sci., 2011; 1(4):249-257.

2. Anonymous. Ministry of Agriculture, New Delhi. Deptt. of MS data collection, 2018.

3. Aslam H, Ansari MA, Khan S, Sana B, Baloch U, Baloch AS. Effect of irrigation scheduling on the growth and harvest index of wheat (Triticum aestivum L.) Verities. Afr. J. Agric. Res., 2014; 6(27):2104-2109.

4. Galavi M, Moghaddam HA. Influence of deficit irrigation during growth stages on water use efficiency (WUE) and production of wheat cultivars under rainfed conditions. Int. Res. J. Applied Basic Sci., 2012; 3:2071-2078.

5. Gautam A, Prasad S, Ambati D, Agarwal D, Jajoo A. Performance of durum wheat genotypes under drought and terminal heat stress conditions in changing climatic conditions. Journal of Botanical Sciences. 2016; 5(3):2531 .

6. Khan N, Naqvi FN. Effect of water stress in bread wheat hexaploids. Curr. Res. J. Biol. Sci., 2011; 3(5):487-498.

7. Mekkei MER, Haggan EL, Eman AMA. Effect of different irrigation regimes on grain yield and quality of some Egyption bread wheat cultivars. J. Agri-food Appl. Sci., 2014; 2(9):275-282.

8. Mobasser HR, Mohammadi GN, Abad HHS, Rigi K. Effect of application of elements and variety on biological and grain yield and harvest index of wheat in Zahak region. J. Bio. Env. Sci., 2014; (5)1:134-139.

9. Ngwako S, Mashiqa PK. The effect of irrigation on time growth and yield of winter wheat (Triticum aestivum L.) Cultivars. Intl. J. Agril. Crop Sci., 2013; 5(9):976-982.

10. Raffi SA, Asaduzzaman. Morpho-physiological and biochemical trait based selection of wheat genotypes for drought tolerance. Bangladesh J. Pl. Breed. Genet., 2015; 28(2):09-16.

11. Reynolds M, Foulkes MJ, Slafer GA, Berry X, Parry MAJ, Snape JW et al. Raising yield potential in wheat. J. Exp. Bot., 2009; 60:1899-1918.

12. Saeidi M, Abdoli M. Effect of drought stress during grain filling on yield and its components, gas exchange variables, and some physiological traits of wheat cultivars. J. Agr. Sci. Tech., 2015; 17:885-898.

13. Siddique MRB, Hamid A, Islam MS. Drought stress effect on yield and yield attributes of wheat. Bangladesh J. Agril. Res., 1999; 24(4):621-628.

14. Samarah NH. Effects of drought stress on growth and yield of barley. Agronomy for Sustainable Development. 2005; 25(1):145-149

15. Sheoran S, Thakur V, Narwal S, Sharma I. Differential activity and expression profile of antioxidant enzymes and physiological changes in wheat (Triticum aestivum L.) Under drought. Appl. Biochem. Biotechnology. 2015; 177:1282-1298.

16. Shrief. performance and drought tolerance of Egyptian Wheat Genotypes. Bioscience Research. 2018; 15(3):2880-2892

17. Siddique MRB, Hamid A, Islam MS. Drought stress effects on water relations of wheat. Bot. Bull. Acad. Sin., 2000; 41:35-39.

18. Taiz L, Zeiger E. Plant Physiology. $4^{\text {th }}$ ed. Sinauer Associates Inc. Publishers, Massachusetts, 2006, 211224.

19. Tas S, Tas B. Some physiological of drought stress in wheat genotypes with different ploidity in Turkiye. World J. Agric. Sci., 2007; 3(2):178-183. 